\title{
Comparative trends in hospitalizations for osteoporotic fractures and other frequent diseases between 2000 and 2008
}

\author{
K. Lippuner • S. Grifone • M. Schwenkglenks • \\ P. Schwab • A. W. Popp • C. Senn • R. Perrelet
}

Received: 7 March 2011 / Accepted: 2 May 2011 /Published online: 28 May 2011

(C) International Osteoporosis Foundation and National Osteoporosis Foundation 2011

\begin{abstract}
Summary In Switzerland, the number, incidence, and cost of acute hospitalizations for major osteoporotic fractures (MOF) and major cardiovascular events (MCE) increased in both women and men between 2000 and 2008, although the mean length of stay (LOS) was significantly reduced. Similar trend patterns were observed for hip fractures and strokes (decrease) and nonhip fractures and acute myocardial infarctions (increase).

Introduction The purpose of this study was to compare the trends and epidemiological characteristics of hospitalizations for MOF and other frequent diseases between years 2000 and 2008 in Switzerland.

Methods Trends in the number, age-standardized incidence, mean LOS, and cost of hospitalized MOF and MCE (acute myocardial infarction, stroke, and heart failure) were compared in women and men aged $\geq 45$ years, based on data from the Swiss Federal Statistical Office.

Results Between 2000 and 2008, the incidence of acute hospitalizations for MOF increased by $3.4 \%$ in women and
\end{abstract}

K. Lippuner $(\bowtie) \cdot$ S. Grifone $\cdot$ A. W. Popp $\cdot$ C. Senn $\cdot$ R. Perrelet Osteoporosis Policlinic, Inselspital,

Bern University Hospital and University of Bern,

CH-3010 Bern, Switzerland

e-mail: kurt.lippuner@insel.ch

M. Schwenkglenks

Institute of Pharmaceutical Medicine/ECPM, University of Basel,

Basel, Switzerland

M. Schwenkglenks

Institute of Social and Preventive Medicine,

Bern, Switzerland

P. Schwab

Swiss Federal Statistical Office,

Neuchâtel, Switzerland
$0.3 \%$ in men. In both sexes, a significant decrease in hip fractures $(-15.0 \%$ and $-11.0 \%)$ was compensated by a concomitant, significant increase in nonhip fractures $(+24.8 \%$ and $+13.8 \%)$. Similarly, the incidence of acute hospitalizations for MCE increased by $4.4 \%$ in women and $8.2 \%$ in men, as an aggregated result from significantly increasing acute myocardial infarctions and significantly decreasing strokes. While the mean LOS in the acute inpatient setting decreased almost linearly between years 2000 and 2008 in all indications, the inpatient costs increased significantly $(p<0.001)$ for MOF $(+30.1 \%$ and $+42.7 \%)$ and $\mathrm{MCE}(+22.6 \%$ and $+47.1 \%)$ in women and men, respectively.

Conclusions Between years 2000 and 2008, the burden of hospitalized osteoporotic fractures to the Swiss healthcare system has continued to increase in both sexes. In women, this burden was significantly higher than that of MCE and the gap widened over time.

Keywords Acute myocardial infarction - Breast cancer . Chronic obstructive pulmonary disease $\cdot$ Clinical spine fracture $\cdot$ Cost $\cdot$ Distal radius fracture $\cdot$ Epidemiology $\cdot$ Heart failure - Hip fracture - Osteoporosis · Proximal humerus fracture $\cdot$ Stroke $\cdot$ Switzerland

\section{Introduction}

Osteoporotic fractures are one of the leading causes of morbidity and mortality in industrialized countries [1]. While virtually any fracture may result as a complication from underlying osteoporosis, "typical" osteoporotic fractures occur at the hip, spine, distal forearm, and proximal humerus. Together, they are traditionally referred to as major osteoporotic fractures (MOF) [1-3]. 
In year 2000, in all women living in Switzerland, the overall incidence of hospitalizations for any osteoporotic fracture was twice as high as that for breast cancer and pooled major cardiovascular events (MCE: acute myocardial infarction, stroke, and heart failure) and almost three times that of chronic obstructive pulmonary disease (COPD) [4]. In Swiss men, the overall incidence of hospitalizations for any osteoporotic fracture was half that of pooled MCE and two thirds that of COPD [4]. As recently published, the age-standardized incidences of hospitalized MOF continued to increase in Swiss women and men aged 45 years and older between the years 2000 and 2007, driven by an increasing number and incidence of hospitalizations for nonhip fractures, although the number of hip fractures was on the decline [5].

The aim of the present analysis was to compare the trends and epidemiological characteristics, in female and male patients aged 45 years or older, of MOF, MCE, COPD, and breast carcinoma (women only) in Switzerland, between the years 2000 and 2008. Predefined parameters of interest were absolute number and incidence of hospitalizations, number of hospital days due to fractures, and cost of hospitalizations.

\section{Methods}

A summary of the data processing flow and relevant data sources is shown in Fig. 1.

\section{Selected ICD-10 codes}

Diseases were selected according to the 10th Edition of the International Classification of Diseases (ICD-10) issued by the World Health Organization [6]. MOF were divided into two categories: hip (ICD-10 codes S72.0 (fracture of the femoral neck), S72.1 (pertrochanteric fracture), and S72.2 (subtrochanteric fracture)) and nonhip. Nonhip fractures were defined as: spine fractures coded as S22.0 (fracture of the thoracic spine), S22.1 (multiple fractures of the thoracic spine), S32.0 (fracture of the lumbar spine), S32.7 (multiple fractures of the lumbar spine), and S32.8 (other fractures of the lumbar spine); fractures of the distal forearm coded as S52.5 (fracture of the distal radius) and S52.6 (combined fracture of the distal radius/ulna); fractures of the proximal humerus coded as S42.2 (fracture of the proximal humerus).

MCE were defined as myocardial infarction coded as I21 (acute myocardial infarction) and I22 (subsequent myocardial infarction); stroke coded as I63 (cerebral infarction), I64 (stroke, not specified as hemorrhage or infarction), I65 (occlusion and stenosis of precerebral arteries, not resulting in cerebral infarction), and I66 (occlusion and stenosis of cerebral arteries, not resulting in cerebral infarction); heart failure coded as I50 (heart failure). COPD were defined as codes J40 (bronchitis, not specified as acute or chronic), J41 (simple and mucopurulent chronic bronchitis), J42 (unspecified chronic bronchitis), J43 (emphysema), J44 (other COPD), J45 (asthma), J46 (status asthmaticus), and
Fig. 1 Data sources and data processing flow. SFSO Swiss Federal Statistical Office, $I C D$ International Classification of Diseases, COPD chronic obstructive pulmonary diseases

\begin{tabular}{|lc|}
\hline \multicolumn{2}{|c|}{ Selected ICD-10 codes (primary diagnosis only) } \\
Major osteoporotic fractures & Major CV events \\
Hip fractures (S72.0, S72.1, S72.2) & Acute myocardial infarction (I21 and I22) \\
Non-hip fractures & Stroke (I63 to I66) \\
Spine fractures & Heart failure (I50) \\
(S22.0, S22.1, S32.0, S32.7, S32.8) & \\
Distal radius fractures (S52.5, S52.6) & COPD (J40 to J47) \\
Proximal humerus fractures (S42.2) & Breast carcinoma (C50) \\
\hline
\end{tabular}

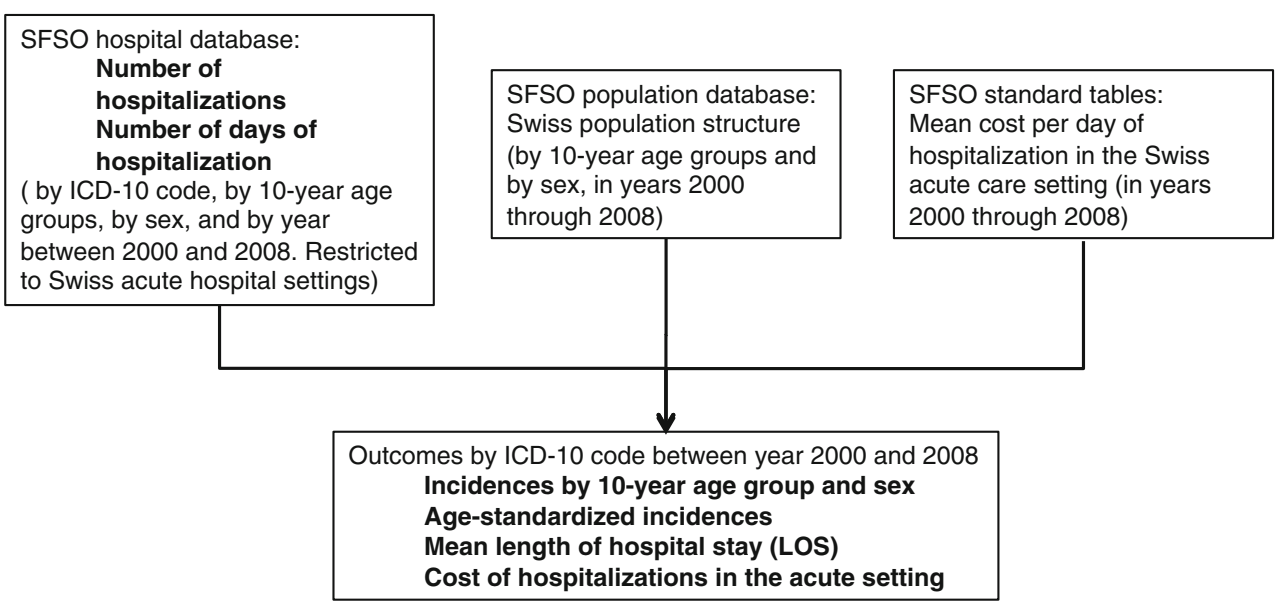


J47 (bronchiectasis). Breast carcinoma corresponded to the ICD-10 code C50 (malignant neoplasm of breast).

\section{Source of hospital statistics}

The administrative and medical statistics database of the Swiss Federal Statistical Office (SFSO) was used. All Swiss acute care hospitals, rehabilitation centers, and specialized clinics are obliged to supply their medical data to the SFSO (age, sex, number of hospitalizations coded according to the ICD-10 requirements, and duration of hospitalization). Data collection is coordinated by the SFSO. Data consistency and quality are ensured at the individual hospital, cantonal, and federal level through the mandatory use of a plausibility testing software developed by the SFSO [7]. This software performs more than 700 plausibility checks on single and pooled variables, including multiple interdependent variable testing and diagnostic code vs. treatment code testing. Inconsistencies and errors indicated in the log files are corrected at the appropriate level (individual hospital for source data, canton for inconsistencies across hospitals, and confederation for inconsistencies across cantons) following a feedback loop. This process ensures low error rates so that the level of plausibility of the reported diagnostic codes exceeds 95\% [8]. Only medical data from Swiss acute hospital settings were retained for the present analysis. Data from rehabilitation centers and specialized clinics were excluded to prevent double counting. The degree of completeness for all records for years 2000 to 2008 was $81.2 \%, 84.9 \%, 95.0 \%, 94.1 \%, 96.2 \%, 98.2 \%, 98.5 \%$, $98.6 \%$, and $98.8 \%$, respectively.

Raw data for the number of hospitalizations for the selected ICD-10 codes and corresponding duration of hospitalization were obtained for women and men aged $\geq 45$ years. Only the primary diagnostic code, i.e., the reason for hospitalization, was considered for each patient to prevent double counting. All raw data were extrapolated pro rata to $100 \%$ to compensate for incomplete coverage and to become representative for Switzerland as a whole.

Data on the Swiss population structure between 2000 and 2008, split by 10-year age groups and sex, were also obtained from the SFSO.

\section{Epidemiological characteristics of hospitalizations}

The age-standardized incidence of hospitalizations, the number of patient-days spent in hospital, and the mean hospital length of stay (LOS) per patient were calculated by 10 -year age groups starting from age 45 onwards and by sex for all selected ICD-10 codes grouped into the categories defined above. Annual crude incidences and $95 \%$ confidence intervals per 100,000 persons in years 2000 to 2008 were calculated by sex and 10-year age groups starting from age 45 onwards. Annual age-standardized incidences per 100,000 persons and 95\% confidence intervals were calculated by sex based on 10-year age groups and adjusted for the age structure of the Swiss population in year 2000 according to a method published earlier [9]. Standardized incidence rate ratios and 95\% confidence intervals were calculated to assess the change in incidence over time. As the goodness-of-fit of tentative Poisson regression models could not be confirmed, related time trends were assessed by fitting gender-specific negative binomial models with ICD-10 code and year as covariates.

The mean hospital LOS was estimated by dividing the total number of patient-days spent in hospital by the number of hospitalizations. The cost of hospitalizations was estimated by multiplying the number of patient-days spent in hospital by the yearly average cost of 1 day in hospital in Swiss Francs (CHF) between 2000 and 2008. The latter was obtained from yearly summary tables derived from the hospital statistics database of the SFSO for each year of analysis and was not inflation adjusted [10]. The effects of time on mean LOS and hospitalization cost were tested for statistical significance by the two-way analysis of variance method. All calculations were done with the statistical software StatsDirect version 2.7.8 (StatsDirect Ltd., Altrincham, Cheshire, UK). Negative binomial regression was performed in Stata/MP version 11.1 (Stata Corporation, College Station, TX, USA).

\section{Results}

Swiss population structure

Between years 2000 and 2008, the number of women and men aged 45 years and older increased by $13.3 \%$ and $17.4 \%$, respectively.

\section{Number of hospitalizations}

Between 2000 and 2008, the absolute number of acute care hospitalizations for MOF in patients aged $\geq 45$ years increased from 13,948 to $16,339(+17.1 \%)$ in women and from 4,670 to $5,634(+20.6 \%)$ in men, essentially driven by nonhip fractures $(+41.3 \%$ and $+34.6 \%$, respectively) (Fig. 2). During the same time, the number of hip fractures decreased from 7,684 to $7,485(-2.6 \%)$ in women and remained stable in men (from 2,538 to $2,765,+1 \%$ ). Similarly, the number of hospitalizations for MCE increased from 9,530 to $11,271(+18.3 \%)$ and from 12,634 to $16,378(+29.6 \%)$ in women and men, respectively. In contrast, the number of hospitalizations for COPD decreased from 5,115 to $4,210(-17.7 \%)$ and from 6,844 to 
Fig. 2 Number of acute care hospitalizations in women (a) and men (b) for selected diseases between years 2000 and 2008
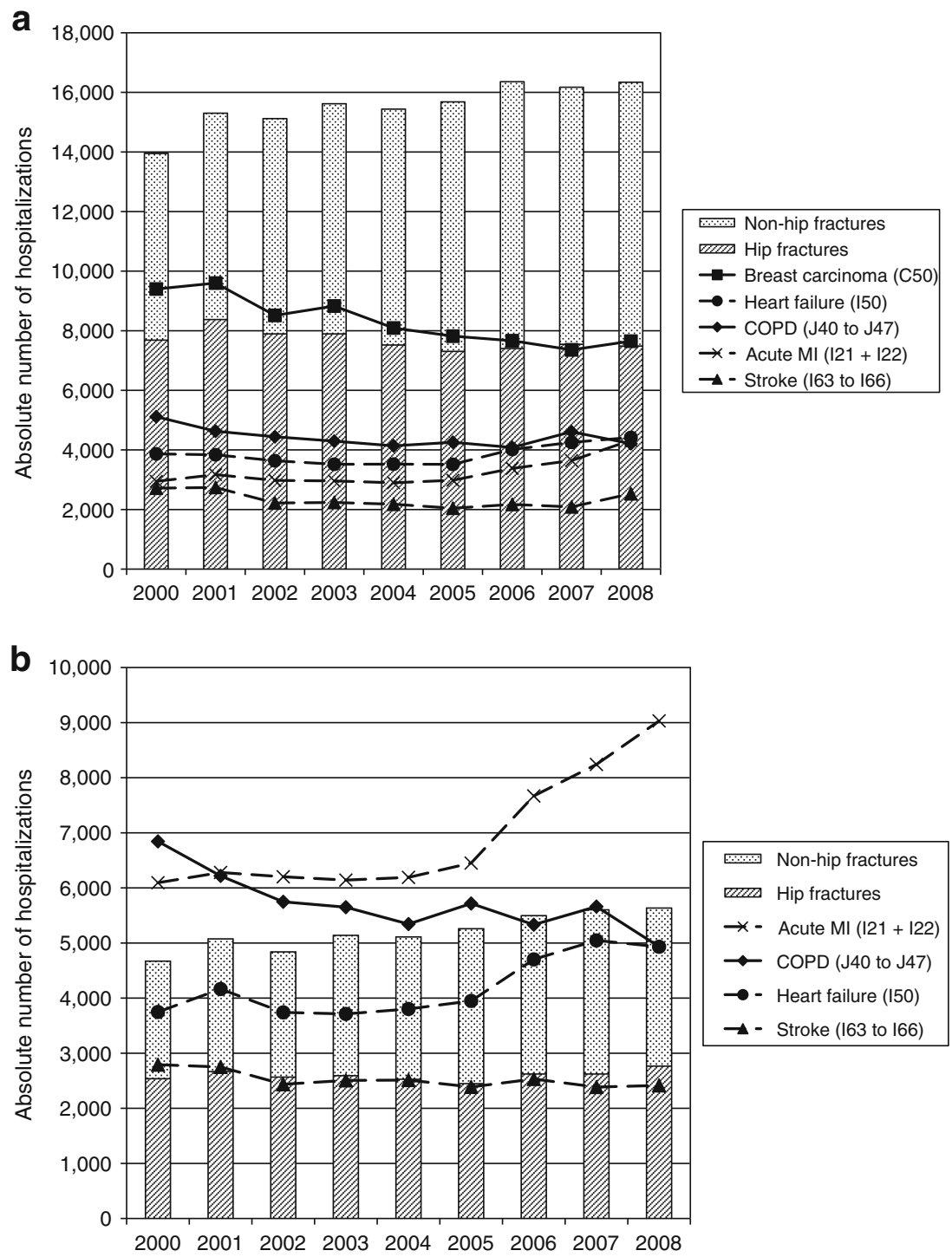

4,934 $(-27.9 \%)$ in women and men, respectively. Finally, the number of hospitalizations for breast cancer decreased from 9,400 to $7,650(-18.6 \%)$ in women.

\section{Age-standardized incidence of hospitalizations}

In women, as shown in Table 1, the age-standardized incidence of hospitalized MOF increased by $3.4 \%$ between 2000 and 2008 ( $p$ for trend, 0.121), the significant decrease in hip fractures $(-15.0 \% ; p$ for trend, $<0.001)$ being fully compensated by a concomitant significant increase in hospitalized nonhip fractures $(+24.8 \%$; $p$ for trend, 0.002). The latter resulted from an increase in hospitalized fractures of the spine $(+32.2 \%$; $p$ for trend, 0.008$)$, the distal radius $(+31.8 \% ; p$ for trend, 0.005$)$, and the proximal humerus $(+10.3 \%, p$ for trend, 0.141). Similarly, the incidence of acute hospitalizations for MCE increased by $4.4 \%$ ( $p$ for trend, 0.558) during the observation period, as an aggre- gated result of a significant increase in acute myocardial infarctions $(+29.5 \%$; $p$ for trend, 0.016) opposed by a significant decrease in hospitalized strokes $(-17.8 \% ; p$ for trend, 0.016), and with no change in the incidence of hospitalizations for heart failure $(+0.8 \% ; p$ for trend, 0.926). Finally, the incidence of hospitalizations for COPD and breast cancer decreased significantly by $27.3 \%$ ( $p$ for trend, 0.016 ) and $28.2 \%$ ( $p$ for trend, $<0.001$ ), respectively.

In men, as shown in Table 1 , the incidence of hospitalized MOF did not change between 2000 and $2008(+0.3 \%$; $p$ for trend, 0.918). The significant reduction in incident hip fractures $(-11.0 \% ; p$ for trend, 0.031 ) was totally offset by a significant increase in nonhip fractures $(+13.8 \% ; p$ for trend, 0.032), mainly driven by fractures of the distal radius $(+29.2 \%$; $p$ for trend, $<0.001)$ and the proximal humerus $(+12.5 \%$; $p$ for trend, 0.295). The incidence of acute hospitalizations for MCE increased significantly by $8.2 \%$ ( $p$ for trend, 0.019 ). 


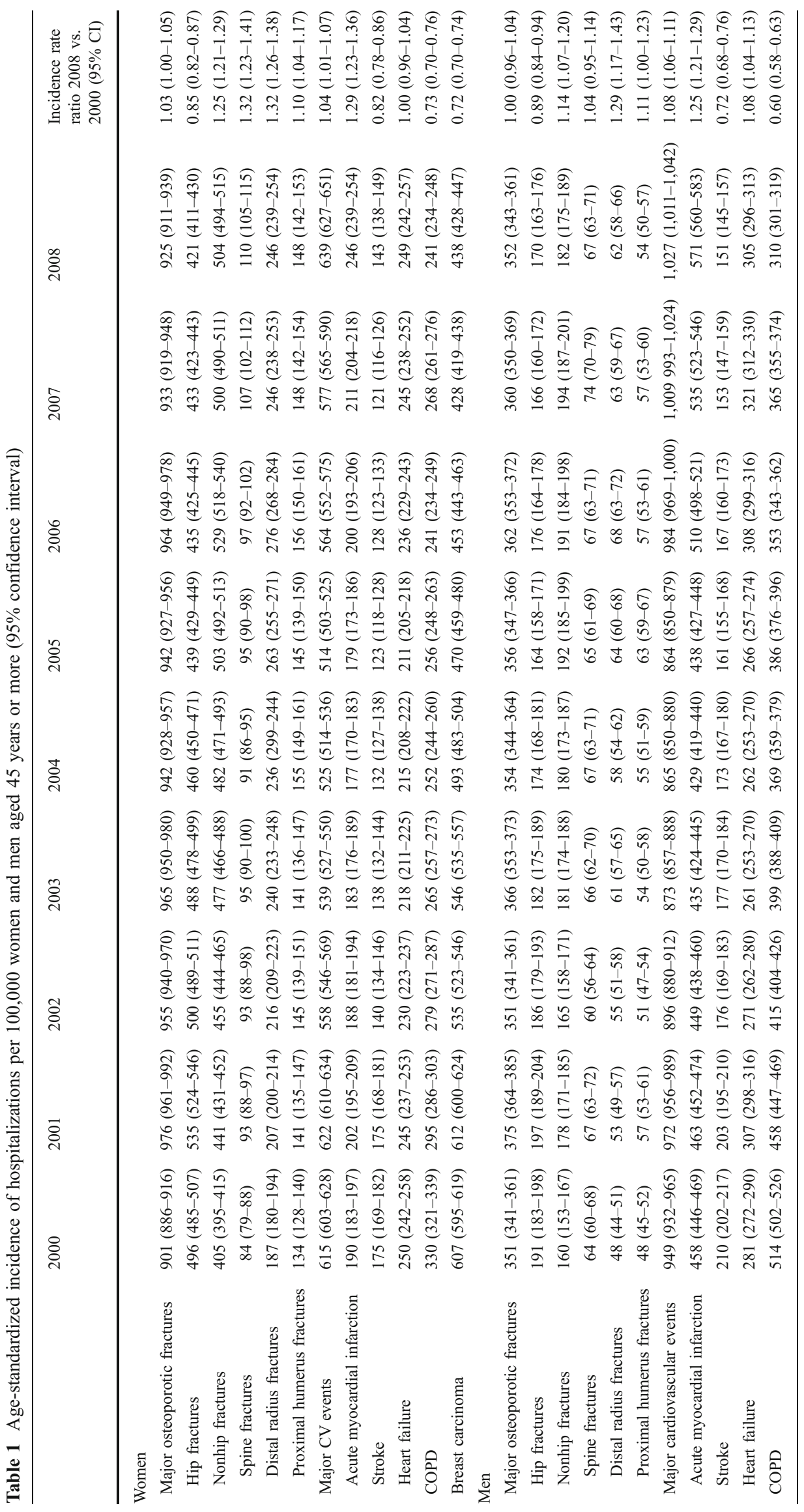


Interestingly, this overall increase resulted from an increase in hospitalizations for myocardial infarctions $(+24.7 \% ; p$ for trend, $<0.001)$ and heart failure $(+8.5 \%$; $p$ for trend, 0.208) opposed by a significant reduction in the incidence of stroke $(-28.1 \% ; p$ for trend, 0.006). Finally, incident hospitalizations for COPD were significantly reduced by $39.7 \%$ ( $p$ for trend, 0.002 ).

The overall pattern of changes in age-standardized incidences was consistent across sexes: decreasing incidences of hospitalizations for hip fractures and stroke, increasing incidences in hospitalizations for nonhip MOF and acute myocardial infarctions, and decreasing incidence in hospitalizations for COPD. The observed increasing or decreasing trends in any disease category went in the same direction and were of similar magnitude in all age groups (Fig. 3).

Patient-days in hospital and mean length of stay

The total number of days spent during the acute hospitalization phase decreased almost linearly between years 2000 and 2008 in all indications of interest and in both sexes. In
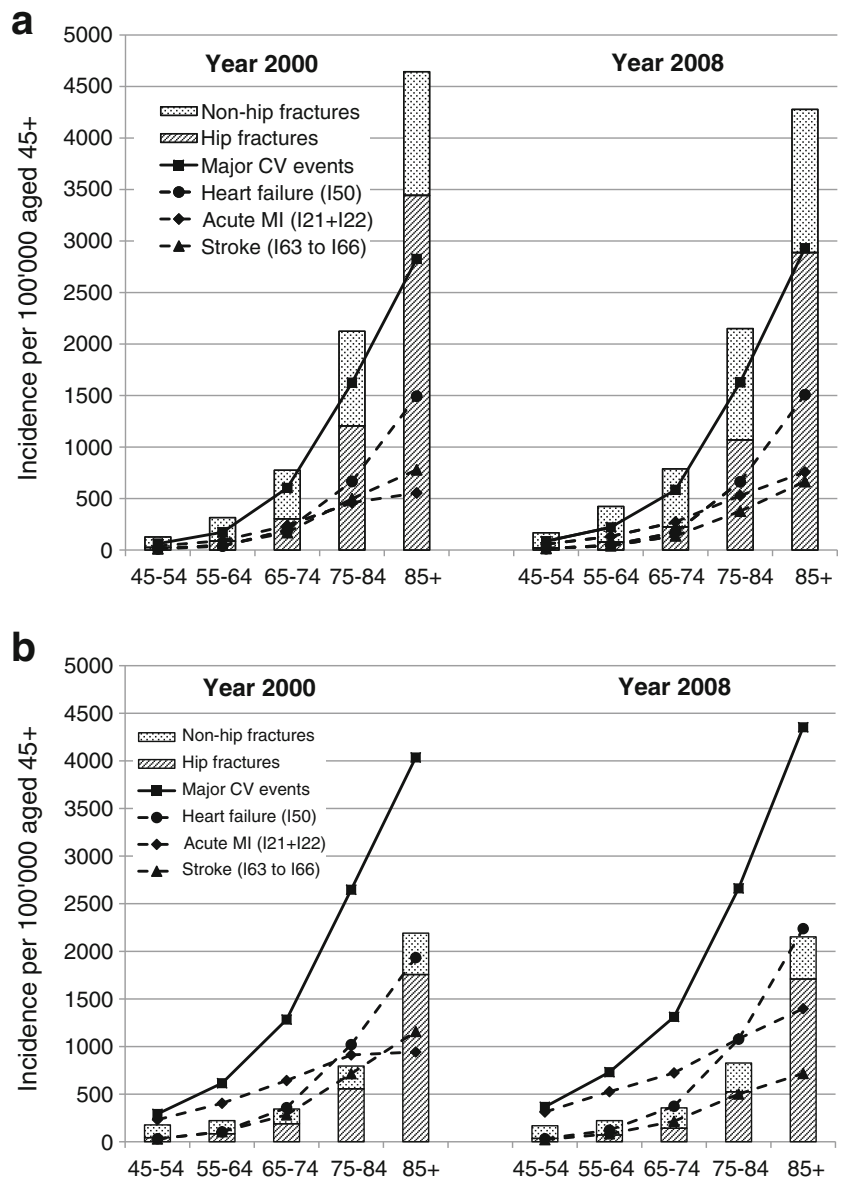

Fig. 3 Age-standardized incidence of hospitalizations per 100,000 in women (a) and men (b) aged 45 years or more by 10-year age groups women, the highest relative decrease was observed for COPD (from 89,554 to 56,099 days, $-37.4 \%$ ), followed by breast carcinoma (from 90,951 to 57,842 days, $-36.4 \%$ ), MCE (from 164,685 to 124,384 days, $-24.5 \%$ ), and MOF (from 224,244 to 179,461 days, $-19.9 \%$ ). Therefore, hospitalization for MOF remains a major contributor of days spent in hospital by women aged 45 years or more, responsible for three times more days of hospitalization than COPD and breast cancer and 1.5 times more days than any MCE.

In men, the highest relative decrease in days spent in hospital was observed for COPD (from 107,329 to $60,663,-43.5 \%$ ) followed by MOF (from 71,477 to $62,828,-12.1 \%$ ) and MCE (from 174,858 to $158,441,-9.4 \%$ ). Therefore, in 2008 , there were as many days spent in hospital by men for COPD, acute myocardial infarction (67,997 days), and heart failure $(57,822$ days $)$ as for MOF.

These considerable reductions in total days spent in hospital for all indications of interest were essentially due to a relevant and statistically significant reduction of the mean LOS as shown in Table 2.

\section{Cost of hospitalizations}

As described above, the mean LOS for patients hospitalized because of fractures decreased substantially between 2000 and 2008. The effect of this was counterbalanced, however, by rising hospital costs: the mean cost per day of hospitalization increased from CHF 996.00 to $\mathrm{CHF}$ $1,618.00$ over the same period, a rise of $62 \%$. The effects on hospitalization costs by selected diagnostic codes are shown in Fig. 4.

In women, hospitalization cost for MOF increased from CHF 223 to 291 million (mio; $+30.1 \%, p<0.001$ for time trend) and from 164 to $201 \mathrm{mio}(+22.6 \%, p<0.001)$ for MCE. The cost of hospitalizations for COPD and breast cancer remained almost unchanged (from CHF 89 to 91 mio, $+1.7 \%, p=0.168$ and from 91 to $94 \mathrm{mio},+3.3 \%, p=$ 0.003 , respectively). Overall, in women in 2008 , the cost of acute hospitalizations was more than threefold higher for MOF than for COPD and breast cancer and 1.5-fold higher than for any MCE. As shown in Fig. 5, osteoporotic fractures contributed to approximately $60 \%$ of the total costs of MOF and MCE in women.

In men, the cost of acute hospitalizations for MOF and MCE between 2000 and 2008 increased by $42.7 \%$ (from CHF 71 to 102 mio, $p<0.001$ for time trend) and by $47.1 \%$ (from CHF 174 to 256 mio, $p<0.001$ ), respectively. Therewhile, the cost of hospitalizations for COPD was reduced by $8.2 \%$ (from CHF 107 to $98 \mathrm{mio}, p=0.078$ ). Overall, in men in 2008, the cost of acute hospitalizations for MOF was comparable to that of COPD, acute myocardial infarction (CHF $110 \mathrm{mio}$ ), and heart failure 
Table 2 Changes in mean hospital LOS (days) in women and men

\begin{tabular}{|c|c|c|c|c|c|c|c|c|c|c|c|}
\hline & 2000 & 2001 & 2002 & 2003 & 2004 & 2005 & 2006 & 2007 & 2008 & $\begin{array}{l}\text { Change } 2008 \\
\text { vs. } 2000(\%)\end{array}$ & $\begin{array}{l}p \text { value for } \\
\text { time effect }\end{array}$ \\
\hline \multicolumn{12}{|l|}{ Women } \\
\hline Major osteoporotic fractures & 16.1 & 15.0 & 14.6 & 13.8 & 12.9 & 12.2 & 11.5 & 11.4 & 11.0 & -31.6 & $<0.001$ \\
\hline Hip fractures & 19.0 & 17.8 & 17.9 & 17.6 & 16.5 & 16.0 & 14.9 & 14.7 & 14.0 & -26.5 & $<0.001$ \\
\hline Nonhip fractures & 12.4 & 11.5 & 11.0 & 9.9 & 9.4 & 8.8 & 8.7 & 8.6 & 8.5 & -32.1 & $<0.001$ \\
\hline Spine fractures & 16.7 & 14.8 & 16.7 & 15.6 & 13.9 & 13.4 & 13.4 & 12.3 & 12.6 & -24.5 & $<0.001$ \\
\hline Distal radius fractures & 8.7 & 8.3 & 7.0 & 6.2 & 6.3 & 5.7 & 5.5 & 5.6 & 5.4 & -38.3 & $<0.001$ \\
\hline Proximal humerus fractures & 15.0 & 14.2 & 13.2 & 12.4 & 11.6 & 11.5 & 11.3 & 10.9 & 10.5 & -30.1 & $<0.001$ \\
\hline Major CV events & 17.3 & 16.1 & 15.4 & 15.2 & 14.6 & 13.4 & 12.7 & 12.0 & 11.0 & -36.1 & $<0.001$ \\
\hline Acute MI & 12.6 & 12.1 & 11.9 & 11.2 & 10.2 & 9.6 & 9.4 & 9.3 & 9.5 & -24.1 & $<0.001$ \\
\hline Stroke & 23.7 & 21.6 & 20.7 & 20.7 & 19.8 & 18.2 & 16.7 & 14.8 & 10.7 & -55.1 & $<0.001$ \\
\hline Heart failure & 16.4 & 15.6 & 15.2 & 15.0 & 15.0 & 13.8 & 13.4 & 13.1 & 12.7 & -22.2 & 0.006 \\
\hline COPD & 17.5 & 16.8 & 16.8 & 15.3 & 14.7 & 13.6 & 13.8 & 13.3 & 13.3 & -23.9 & $<0.001$ \\
\hline Breast carcinoma & 9.7 & 9.2 & 8.9 & 8.7 & 8.2 & 7.7 & 7.7 & 7.6 & 7.6 & -21.9 & $<0.001$ \\
\hline \multicolumn{12}{|l|}{ Men } \\
\hline Major osteoporotic fractures & 15.3 & 14.0 & 13.9 & 13.3 & 12.5 & 11.5 & 11.7 & 11.2 & 11.2 & -27.1 & $<0.001$ \\
\hline Hip fractures & 18.8 & 17.2 & 17.4 & 16.7 & 15.9 & 15.2 & 15.1 & 14.4 & 13.8 & -27.0 & $<0.001$ \\
\hline Nonhip fractures & 11.1 & 10.5 & 10.0 & 9.7 & 9.2 & 8.3 & 8.6 & 8.4 & 8.6 & -22.1 & $<0.001$ \\
\hline Spine fractures & 13.9 & 13.5 & 13.7 & 12.3 & 11.5 & 10.5 & 11.3 & 10.3 & 11.0 & -20.9 & $<0.001$ \\
\hline Distal radius fractures & 6.0 & 6.1 & 5.5 & 5.7 & 5.3 & 5.3 & 5.0 & 4.8 & 5.3 & -12.0 & 0.184 \\
\hline Proximal humerus fractures & 12.4 & 11.1 & 10.4 & 11.1 & 10.4 & 9.2 & 9.6 & 10.0 & 9.6 & -23.1 & 0.004 \\
\hline Major CV events & 13.8 & 13.1 & 12.0 & 11.7 & 11.4 & 10.5 & 10.2 & 9.8 & 9.7 & -30.1 & $<0.001$ \\
\hline Acute MI & 9.4 & 9.3 & 8.6 & 8.2 & 8.0 & 7.5 & 7.4 & 7.3 & 7.5 & -19.8 & $<0.001$ \\
\hline Stroke & 23.0 & 20.8 & 19.2 & 18.6 & 18.1 & 15.6 & 15.3 & 13.7 & 13.5 & -41.3 & $<0.001$ \\
\hline Heart failure & 14.3 & 13.9 & 12.9 & 12.7 & 12.6 & 12.2 & 12.0 & 11.9 & 11.7 & -17.7 & 0.225 \\
\hline COPD & 15.7 & 15.7 & 14.4 & 14.4 & 13.6 & 12.9 & 13.0 & 12.5 & 12.3 & -21.6 & $<0.001$ \\
\hline
\end{tabular}

(CHF 94 mio). As shown in Fig. 5, osteoporotic fractures represented approximately $30 \%$ of the total costs of MOF and MCE in men.

\section{Discussion}

The present analysis reports the epidemiological characteristics of hospitalizations in the acute care setting of patients with MOF, MCE, COPD, or breast carcinoma in Switzerland between years 2000 and 2008. For the first time, time trends were established by using a nationwide database of medical records following an identical methodology, which ensures the highest possible homogeneity in results and should allow for comparisons with only little potential for bias. In this analysis, the age-standardized rates of hospitalizations for MOF and MCE increased during the observation period, driven by an increase in nonhip fractures and acute myocardial infarctions, respectively. In addition, the mean hospital LOS decreased significantly in all indications of interest, the cost effect of which was offset by the increase in cost per day of hospitalization. Overall, the total cost of hospitalizations, which aggregates the cost effect of the number of hospitalizations, mean hospital LOS, and cost per day of hospitalization, increased by $30 \%$ and $43 \%$ for MOF and $23 \%$ and $47 \%$ for MCE in women and men, respectively. The total cost incurred by hospitalizations for COPD and breast cancer remained virtually unchanged. During the same time, hospitalizations for hip fractures, stroke, COPD, and breast cancer decreased significantly. Reported changes followed similar patterns in both sexes, although of different orders of magnitude.

In Swiss women, during the 9 years of observation, osteoporosis and its complications, as measured by the characteristics of hospitalizations for MOF, remained a key contributor to the economic burden of healthcare. As confirmed by the present analysis, hip fractures seem to decline in Swiss women, which confirm earlier findings from our group [5] and others [11, 12]. This possible reversal of a secular trend is consistent with reports from other countries such as USA [13-15], Canada [16, 17], Japan [18], Finland [19], and Denmark [20]. Similar observations were made in neighbor countries such as France [21] and Austria [22] but not in Italy [23] and 
Fig. 4 Cost of acute hospitalizations in women (a) and men (b) aged 45 years and older (mio CHF)
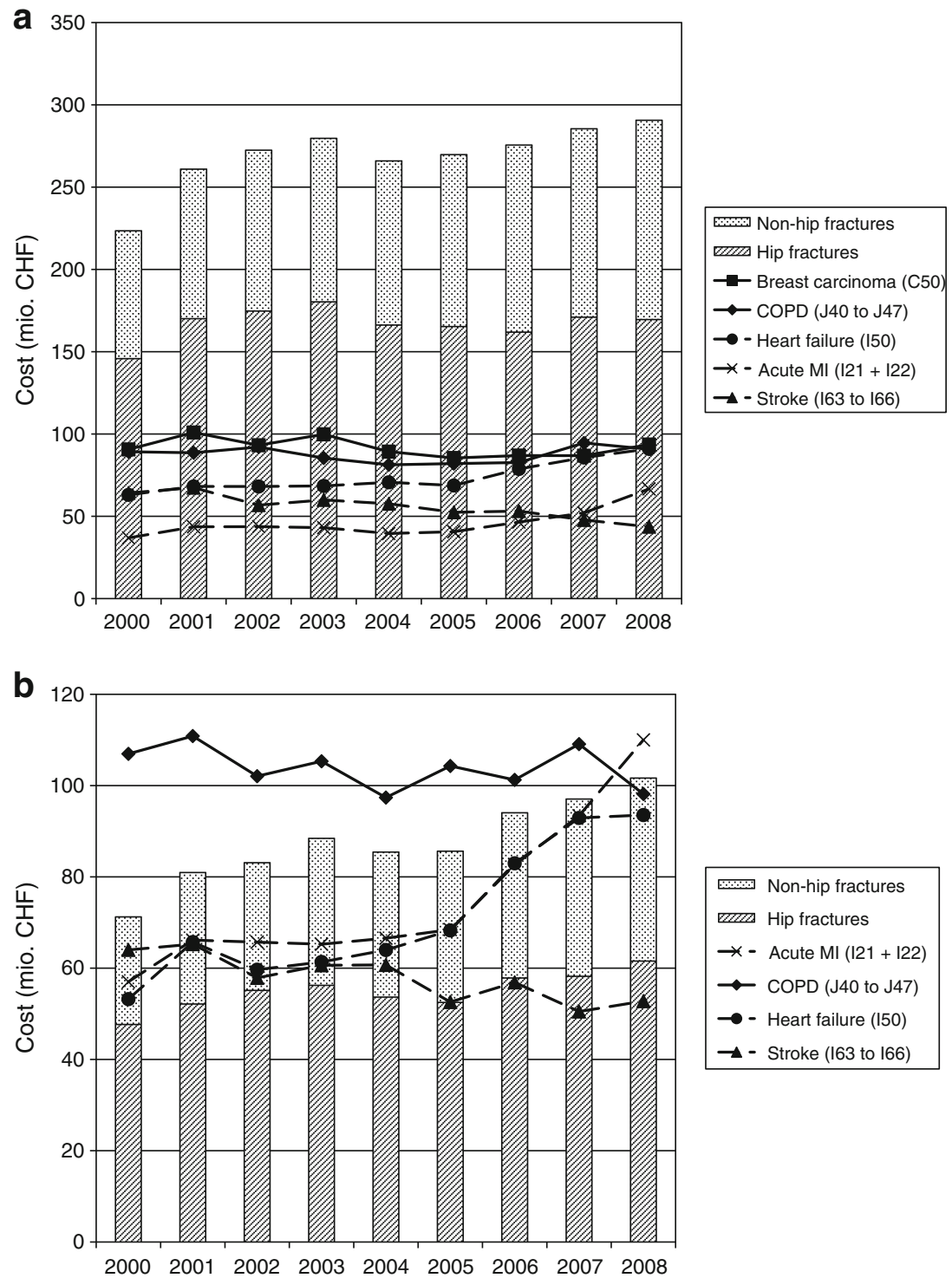

Germany [24, 25]. In contrast, the incidence of hospitalizations for nonhip fractures steadily increased between years 2000 and 2008 in Swiss women. This effect was observed for all localizations of nonhip fractures (spine, distal radius, and proximal humerus). Possible explanations will remain speculative and were detailed elsewhere [5]. We are not aware of other publications confirming or infirming this observation, with the exception of earlier reports from Finland which indicated that low-trauma knee [26], ankle [27], and distal humeral [28] fractures may be stabilizing or even declining in this country. Importantly, the present analysis reports hospitalized fractures only. Whether the total incidence of hospitalized and nonhospitalized nonhip fractures has increased cannot be concluded from the present data. However, as ongoing healthcare reform efforts have led to a global shift from inpatient to outpatient care [29], the matter deserves further investigation. The age- standardized incidence of hospitalizations for hip and nonhip fractures in men followed a similar pattern as in women but with a lower magnitude of change.

Interestingly, in women, the incidence of hospitalizations for acute myocardial infarctions increased strongly, while they stagnated for heart failure and decreased for stroke, with a similar pattern in men. Although a milestone regarding the characteristics of acute coronary syndromes in the real world was achieved already in 1997 by launching the Acute Myocardial Infarction in Switzerland (AMIS) and AMIS Plus registry [30], we are not aware of previously published trend patterns for hospitalized MCE in Switzerland. An increasing number of publications report the association between cardiovascular diseases and osteoporotic fractures. Among the very first indices in this regard, a Swedish registry collected 273,288 strokes and 24,666 fractures, of which 14,263 were hip fractures, 

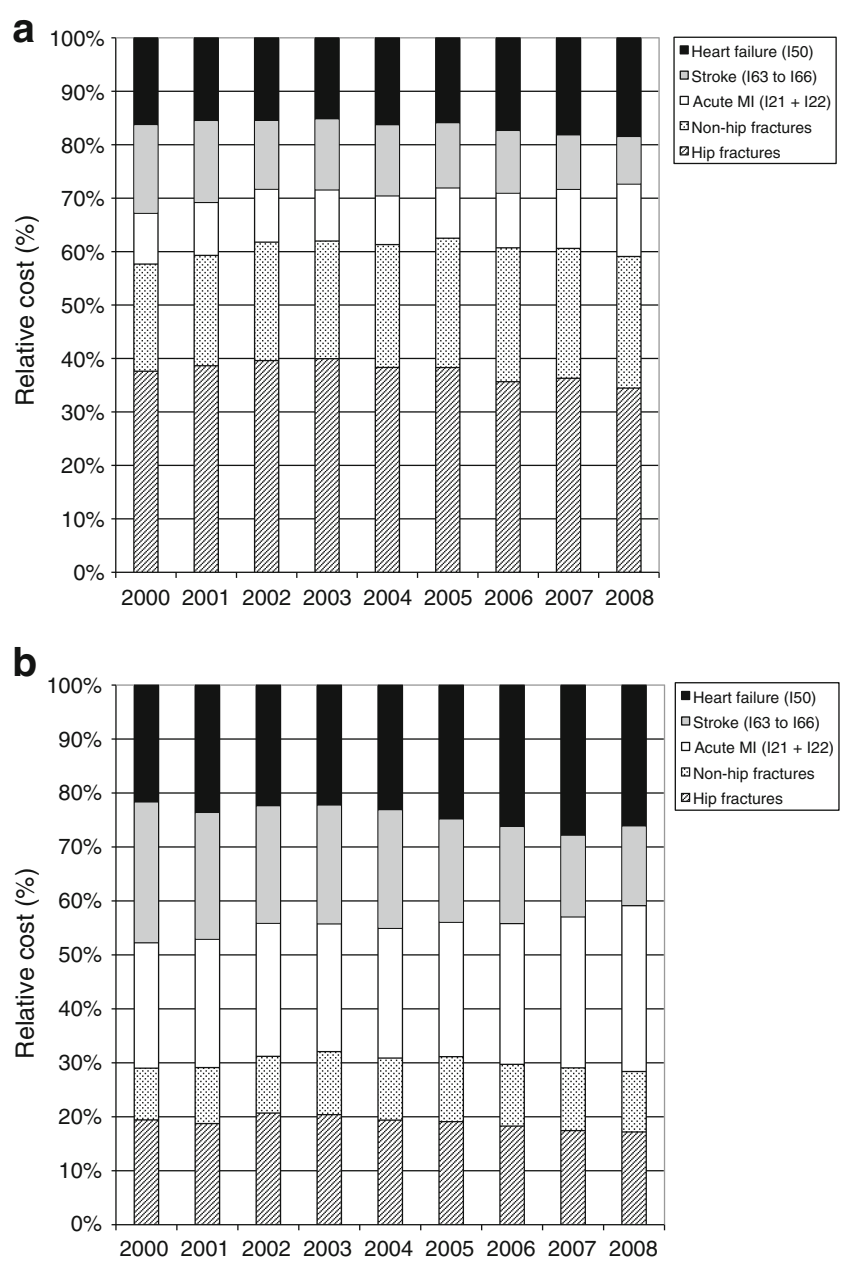

Fig. 5 Relative cost contribution of MOF and MCE in women (a) and men (b) aged 45 years and older between years 2000 and 2008

between 1987 and 1996 and concluded that, after a hospitalization for stroke, there was a more than sevenfold increase in fracture risk during the first year after hospitalization for stroke [31]. In 2007, Sennerby et al. showed that women with cardiovascular disease had a substantially increased risk of hip fracture, suggesting possible common pathologic pathways for osteoporotic fractures and cardiovascular diseases [32]. In addition, in a cohort of 31,936 Swedish twins born in 1914-1944 followed up from the age of 50 years, the same authors reported that a diagnosis of cardiovascular disease was significantly associated with risk of subsequent hip fracture within the same individual and in his or her co-twin without index diagnosis [33]. In the present analysis, we report an increasing incidence of both MOF and major cardiovascular diseases between 2000 and 2008 in Switzerland, which is consistent with a possible association between both disease entities. We also report decreasing trends in hip fractures and stroke and increasing trends in nonhip fractures and acute myocardial infarctions, which raises the question whether the supposed association between osteoporosis and cardiovascular diseases might be more multifaceted than suggested to date.

The present analysis based on data of the medical database of the SFSO did not allow for identification of potential underlying associations or causes for the reported time trends. The earlier mentioned prospective AMIS Plus registry collects 230 variables of patients hospitalized for acute coronary syndromes in 76 Swiss hospitals participating on a voluntary basis. If a similar registry existed for osteoporotic fractures, ideally in the same or a subset of the AMIS hospitals, database linkages could offer a unique opportunity for exploring further the associations between fractures and cardiovascular diseases. Furthermore, both fractures and MCE often lead to patient discharge to a rehabilitation center. Here again, a fracture registry combined with AMIS Plus could possibly contribute to identify common prognostic factors and to improve outcomes.

The present analysis reports hospitalized clinical events only. It is well known that many osteoporotic fractures are treated in the outpatient setting. Based on data from the Swiss nationwide survey in the OsteoCare cohort, which included 3,667 consecutive women and men aged 50 years and older and presenting to the emergency ward of participating hospitals with a fragility fracture [34], virtually all hip fractures, approximately half of proximal humerus fractures, one third of distal radius fractures, and one fourth of clinical spine fractures were hospitalized in Switzerland [3]. These observations are broadly consistent with reports from other countries [35]. On the other hand, as many as $25 \%$ of all coronary events are lethal before the patient reaches the hospital, a proportion that continues to increase as a result of the decline in in-hospital deaths observed during the past decades [36]. Therefore, the proposed comparisons across diseases should be considered as restricted to hospitalizations only.

We reported on MOF, i.e., of a subset of all hospitalized osteoporotic fractures. Achieving completeness would have required the very cumbersome use of osteoporosis attribution rates, as was exemplified earlier $[4,37]$. Using the present approach, MOF were estimated to represent only $77 \%$ and $72 \%$ of attributable fractures in women and men in year 2000, respectively [3-5]. Therefore, absolute numbers, incidences, hospital days, and related costs reported in the analysis should be considered conservative. Data completeness was $81.2 \%$ and $84.9 \%$ in years 2000 and 2001, respectively, and exceeded $95.0 \%$ thereafter. The extrapolation to $100 \%$ could have induced a selection bias when comparing year 2008 with 2000. However, the trends observed over the 9 years were consistent year over year in all diseases of interest and no outliers were identified.

Of note, the total cost estimate for osteoporotic fractures (CHF 393 mio) reported in the present analysis refers to 
direct medical costs of acute hospitalizations for MOFs only. The total direct medical cost of all hospitalized osteoporotic fractures can be extrapolated to CHF 524 mio, to which the inpatient rehabilitation and nursing home costs should be added. These represent approximately $50 \%$ of the total cost of the inpatient management of osteoporotic fractures, as already published earlier by our group [38], so that the total cost of treating hospitalized osteoporotic fractures in Switzerland exceeded CHF 1 billion in 2008. Cost estimates calculated in the present analysis are based on the nationwide average real cost per day of hospitalization and not on discipline-specific costs or diagnosis-related groups (DRGs). Therefore, cost estimates should be regarded as approximations, until the introduction of the DRG system will allow more precise estimates.

Finally, we did not attempt a projection based on observed trends. According to demographic scenarios from the SFSO, the Swiss population older than 65 years of age will almost have doubled in 2050 [39]. Therefore, assuming that the current time trends remain unchanged, the declining incidence of hip fractures and strokes will not be sufficient to reduce the absolute number of hospitalizations for these diseases in the coming decades. A representative cohort or a fracture registry linked to the AMIS Plus registry would contribute to better anticipation of future trends and allow for monitoring the effects of public health measures aimed at reducing fracture and cardiovascular risk.

In conclusion, between years 2000 and 2008, the burden of hospitalized osteoporotic fractures to the Swiss healthcare system has continued to increase in both sexes. In women, this burden was significantly higher than that of MCE and the gap between both disease groups widened over time. More research is warranted to better understand the causal drivers behind these trends.

Acknowledgements This work was supported by an unrestricted research grant from MSD Switzerland AG. The sponsor had no influence on the design, analysis, or interpretation of the data. We are grateful to Dr. Philippe Kress, Kressmed, Glattbrugg, Switzerland for his contribution to data analysis and his critical review of the manuscript.

\section{Conflicts of interest None.}

\section{References}

1. Kanis JA, Burlet N, Cooper C, Delmas PD, Reginster JY, Borgstrom F, Rizzoli R (2008) European guidance for the diagnosis and management of osteoporosis in postmenopausal women. Osteoporos Int 19:399-428

2. Lippuner K, Johansson H, Kanis JA, Rizzoli R (2010) FRAX assessment of osteoporotic fracture probability in Switzerland. Osteoporos Int 21:381-389
3. Lippuner K, Johansson H, Kanis JA, Rizzoli R (2009) Remaining lifetime and absolute 10-year probabilities of osteoporotic fracture in Swiss men and women. Osteoporos Int 20:1131-1140

4. Lippuner K, Golder M, Greiner R (2005) Epidemiology and direct medical costs of osteoporotic fractures in men and women in Switzerland. Osteoporos Int 16(Suppl 2):S8-S17

5. Lippuner K, Popp AW, Schwab P, Gitlin M, Schaufler T, Senn C, Perrelet R (2010) Fracture hospitalizations between years 2000 and 2007 in Switzerland: a trend analysis. Osteoporos Int (in press)

6. World Health Organization (2007) International statistical classification of diseases and related health problems, 10th revision. Online version 2007. Available at http://apps.who.int/classifications/ apps/icd/icd10online/. Accessed 12 January 2011

7. Swiss Federal Statistical Office (2009) Plausibility of medical statistics: the MedPlaus 5.0 concept. Available at http://www. freudiger.com/download/mp5_konzept_D_20091029.pdf. Accessed October 2010

8. Swiss Federal Statistical Office (2004) Data quality evaluation of medical statistics in 1998-2001. Available at http://www.bfs.admin. $\mathrm{ch} / \mathrm{bfs} /$ portal/de/index/themen/14/22/publ.html?publicationID=1306. Accessed October 2010

9. Lewinnek GE, Kelsey J, White AA 3rd, Kreiger NJ (1980) The significance and a comparative analysis of the epidemiology of hip fractures. Clin Orthop Relat Res (152):35-43

10. Swiss Federal Statistical Office (2010) Hospital statistics between year 2000 and year 2008. Available at http://www.bfs.admin.ch/ bfs/portal/de/index/themen/14/03/01/data/01.html and http://www. bfs.admin.ch/bfs/portal/de/index/themen/14/03/01/key/01.html. Accessed 12 January 2011

11. Chevalley T, Guilley E, Herrmann FR, Hoffmeyer P, Rapin CH, Rizzoli R (2007) Incidence of hip fracture over a 10-year period (1991-2000): reversal of a secular trend. Bone 40:1284-1289

12. Guilley E, Chevalley T, Herrmann F, Baccino D, Hoffmeyer P, Rapin CH, Rizzoli R (2008) Reversal of the hip fracture secular trend is related to a decrease in the incidence in institutiondwelling elderly women. Osteoporos Int 19:1741-1747

13. Melton LJ 3rd, Kearns AE, Atkinson EJ, Bolander ME, Achenbach SJ, Huddleston JM, Therneau TM, Leibson CL (2009) Secular trends in hip fracture incidence and recurrence. Osteoporos Int 20:687-694

14. Gehlbach SH, Avrunin JS, Puleo E (2007) Trends in hospital care for hip fractures. Osteoporos Int 18:585-591

15. Brauer CA, Coca-Perraillon M, Cutler DM, Rosen AB (2009) Incidence and mortality of hip fractures in the United States. Jama 302:1573-1579

16. Leslie WD, O'Donnell S, Jean S, Lagace C, Walsh P, Bancej C, Morin S, Hanley DA, Papaioannou A (2009) Trends in hip fracture rates in Canada. Jama 302:883-889

17. Jaglal S (2007) Falling hip fracture rates. J Bone Miner Res 22:1098, Author reply 1099

18. Orimo H, Yaegashi Y, Onoda T, Fukushima Y, Hosoi T, Sakata K (2009) Hip fracture incidence in Japan: estimates of new patients in 2007 and 20-year trends. Arch Osteoporos 4:71-77

19. Kannus P, Niemi S, Parkkari J, Palvanen M, Vuori I, Jarvinen M (2006) Nationwide decline in incidence of hip fracture. J Bone Miner Res 21:1836-1838

20. Abrahamsen B, Vestergaard P (2010) Declining incidence of hip fractures and the extent of use of anti-osteoporotic therapy in Denmark 1997-2006. Osteoporos Int 21:373-380

21. Maravic M, Taupin P, Landais P, Roux C (2011) Change in hip fracture incidence over the last 6 years in France. Osteoporos Int 22:797-801

22. Dimai HP, Svedbom A, Fahrleitner-Pammer A, Pieber T, Resch H, Zwettler E, Chandran M, Borgstrom F (2011) Epidemiology of 
hip fractures in Austria: evidence for a change in the secular trend. Osteoporos Int 22:685-692

23. Piscitelli P, Gimigliano F, Gatto S et al (2010) Hip fractures in Italy: 2000-2005 extension study. Osteoporos Int 21:13231330

24. Wildner M, Clark DE (2001) Hip fracture incidence in East and West Germany: reassessment ten years after unification. Osteoporos Int 12:136-139

25. Icks A, Haastert B, Wildner M, Becker C, Meyer G (2008) Trend of hip fracture incidence in Germany 1995-2004: a populationbased study. Osteoporos Int 19:1139-1145

26. Kannus P, Niemi S, Parkkari J, Sievanen H, Palvanen M (2009) Declining incidence of low-trauma knee fractures in elderly women: nationwide statistics in Finland between 1970 and 2006. Osteoporos Int 20:43-46

27. Kannus P, Palvanen M, Niemi S, Parkkari J, Jarvinen M (2008) Stabilizing incidence of low-trauma ankle fractures in elderly people Finnish statistics in 1970-2006 and prediction for the future. Bone 43:340-342

28. Palvanen M, Kannus P, Niemi S, Parkkari J (2009) Secular trends in distal humeral fractures of elderly women: nationwide statistics in Finland between 1970 and 2007. Bone 46:13551358

29. Swiss Health Observatory OBSAN (2004) 5-year comparison of the inpatient and outpatient costs between 1999 and 2003. Available at http://www.obsan.admin.ch/bfs/obsan/de/index/05/ 01.Document.105404.pdf. Accessed 16 June 2010

30. Radovanovic D, Erne P (2010) AMIS Plus: Swiss registry of acute coronary syndrome. Heart 96:917-921
31. Kanis J, Oden A, Johnell O (2001) Acute and long-term increase in fracture risk after hospitalization for stroke. Stroke 32:702-706

32. Sennerby U, Farahmand B, Ahlbom A, Ljunghall S, Michaelsson $\mathrm{K}$ (2007) Cardiovascular diseases and future risk of hip fracture in women. Osteoporos Int 18:1355-1362

33. Sennerby U, Melhus H, Gedeborg R, Byberg L, Garmo H, Ahlbom A, Pedersen NL, Michaelsson K (2009) Cardiovascular diseases and risk of hip fracture. Jama 302:1666-1673

34. Suhm N, Lamy O, Lippuner K (2008) Management of fragility fractures in Switzerland: results of a nationwide survey. Swiss Med Wkly 138:674-683

35. Tarantino U, Capone A, Planta M et al (2010) The incidence of hip, forearm, humeral, ankle, and vertebral fragility fractures in Italy: results from a 3-year multicenter study. Arthritis Res Ther 12:R226

36. Dudas K, Lappas G, Stewart S, Rosengren A (2011) Trends in out-of-hospital deaths due to coronary heart disease in Sweden (1991 to 2006). Circulation 123:46-52

37. Lippuner K, von Overbeck J, Perrelet R, Bosshard H, Jaeger P (1997) Incidence and direct medical costs of hospitalizations due to osteoporotic fractures in Switzerland. Osteoporos Int 7:414 425

38. Schwenkglenks M, Lippuner K, Hauselmann HJ, Szucs TD (2005) A model of osteoporosis impact in Switzerland 2000 2020. Osteoporos Int 16:659-671

39. Bundesamt für Statistik. Szenarien zur Bevölkerungsentwicklung der Schweiz 2005-2050. Available at http://www.bfs.admin.ch/ bfs/portal/de/index/news/publikationen.Document.83713.pdf. Accessed 14 May 2008 